Wilfrid Laurier University

Scholars Commons @ Laurier

Physics and Computer Science Faculty

Publications

Physics and Computer Science

1994

\title{
Harmonic Synchronization in Resistively Coupled Josephson Junctions
}

James A. Blackburn

Wilfrid Laurier University, jabjabjab@cogeco.ca

Niels Grønbech-Jensen

Los Alamos National Laboratory

H.J.T. Smith

University of Waterloo

Follow this and additional works at: https://scholars.wlu.ca/phys_faculty

\section{Recommended Citation}

Blackburn, James A.; Grønbech-Jensen, Niels; and Smith, H.J.T., "Harmonic Synchronization in Resistively Coupled Josephson Junctions" (1994). Physics and Computer Science Faculty Publications. 59.

https://scholars.wlu.ca/phys_faculty/59

This Article is brought to you for free and open access by the Physics and Computer Science at Scholars Commons @ Laurier. It has been accepted for inclusion in Physics and Computer Science Faculty Publications by an authorized administrator of Scholars Commons @ Laurier. For more information, please contact scholarscommons@wlu.ca. 


\title{
Harmonic synchronization in resistively coupled Josephson junctions
}

\author{
James A. Blackburn \\ Department of Physics and Computing, Wilfrid Laurier University, Waterloo, Ontario N2L 3C5, Canada \\ Niels Gronbech-Jensen \\ Theoretical Division, Los Alamos National Laboratory, Los Alamos, New Mexico 87545 \\ H. J. T. Smith \\ Department of Physics, University of Waterloo, Waterloo, Ontario N2L 3G1, Canada
}

(Received 2 September 1993; accepted for publication 8 December 1993)

\begin{abstract}
The oscillations of two resistively coupled Josephson junctions biased only by a single dc current source are shown to lock harmonically in a 1:2 mode over a significant range of bias current, even when the junctions are identical. The dependence of this locking on both junction and coupling parameters is examined, and it is found that, for this particular two-junction configuration, 1:1 locking can never occur, and also that a minimum coupling coefficient is needed to support harmonic locking. Some issues related to subharmonic locking are also discussed.
\end{abstract}

\section{INTRODUCTION}

The study of interacting pairs of Josephson junctions has a long history, reaching back at least two decades. Interest in these configurations stems in part from their fundamental role in quantum interference devices and superconducting electronics. Many biasing schemes and coupling mechanisms have been examined, ${ }^{1,2}$ but the most elemental arrangement consists of two junctions connected in series, with an external shunt impedance. Again, this shunt impedance can be imagined in endless variations, from purely resistive $e^{3-6}$ to reactive. ${ }^{6-10}$ A simple inductive shunt ${ }^{11,12}$ corresponds to an ideal two-junction interferometer.

For probing the effects of coupling on shunted pairs of junctions, the usual arrangement has been to supply separate bias currents to each junction. Characteristic curves (e.g., Fig. 1 of Ref. 4 and Fig. 2 of Ref. 13) could then be generated by fixing the bias current in one junction at some set value, and then monitoring both junction voltages as the bias current through the second junction was slowly ramped up from zero. At some point in the ramping process, the two characteristics cross or, more precisely, they snap together over some finite range of the swept bias. Within this bias interval the junction oscillations are fully synchronized (locked). This region of 1:1 locking has received much attention in the literature, although harmonic ${ }^{13,14}$ and subharmonic ${ }^{15}$ locking zones have also been discussed.

In a recent paper, ${ }^{16}$ we considered the dynamics of a current-biased Josephson junction loaded by a transmission line which was itself terminated by a second Josephson junction. It was found that the behavior of this configuration was dominated by the purely resistive component of the interjunction coupling, and that the reactive elements of the transmission line produced relatively small additional features that decorated the current-voltage characteristics. This has led us to reconsider the simplest geometry-a pair of resistively coupled Josephson junctions-in order to clarify the origins of the dominant underlying structure in the transmission line problem.

\section{HARMONIC LOCKING: IDENTICAL JUNCTIONS}

The particular circuit to be considered here is indicated schematically in Fig. 1(a) together with an equivalent representation, Fig. 1(b), in which the rearrangement emphasizes the shunting role of the coupling resistance. Although only a single bias source is present, it can be useful to regard this as if it were a two-bias circuit, but with one current set to zero.

As discussed in Ref. 16, the equations governing this system are

$$
I_{\text {bias }}^{*}=(1+\alpha) \dot{\phi}_{A}-(\alpha) \dot{\phi}_{B}+\sin \left(\phi_{A}\right)
$$

and

$$
0=(1+\alpha) \dot{\phi}_{B}-(\alpha) \dot{\phi}_{A}+\sin \left(\phi_{B}\right),
$$

where $\phi_{A}$ and $\phi_{B}$ are the junction phases, $\alpha \equiv R_{J} / R$ is a coupling coefficient, $\Gamma_{\text {bias }}^{*}$ is the input bias current normalized to the junction critical currents $I_{c}$, and overdots denote derivatives with respect to dimensionless time [units of $\left.\hbar /\left(2 e I_{c} R_{j}\right)\right]$.

All results are based on numerical solutions of this pair of equations using time increments of $\Delta t=0.01$ or 0.001 . This gave approximately 1000 or more steps per cycle of the phase oscillations. Time averages $\left\langle d \phi_{A} / d t\right\rangle$ and $\left\langle d \phi_{B} / d t\right\rangle$ were accumulated over several thousand cycles of $d \phi / d t$. All computations were performed in double precision.

Typical $I_{\text {bias }}^{*}$ vs $\langle d \phi / d t\rangle$ curves for the two junctions with $\alpha=2.00$ are shown in Fig. 2. Our attention in this article will be somewhat focused on the region of these characteristics between the dotted lines; Ref. 16 was concerned primarily with bias currents above the upper dotted line. It is interesting to compare the present numerical results (Fig. 2) with purely experimental data taken from a rather elegant integrated thin film realization of the shunted junction arrangement [Fig. 2(a) in Ref. 5, re- 


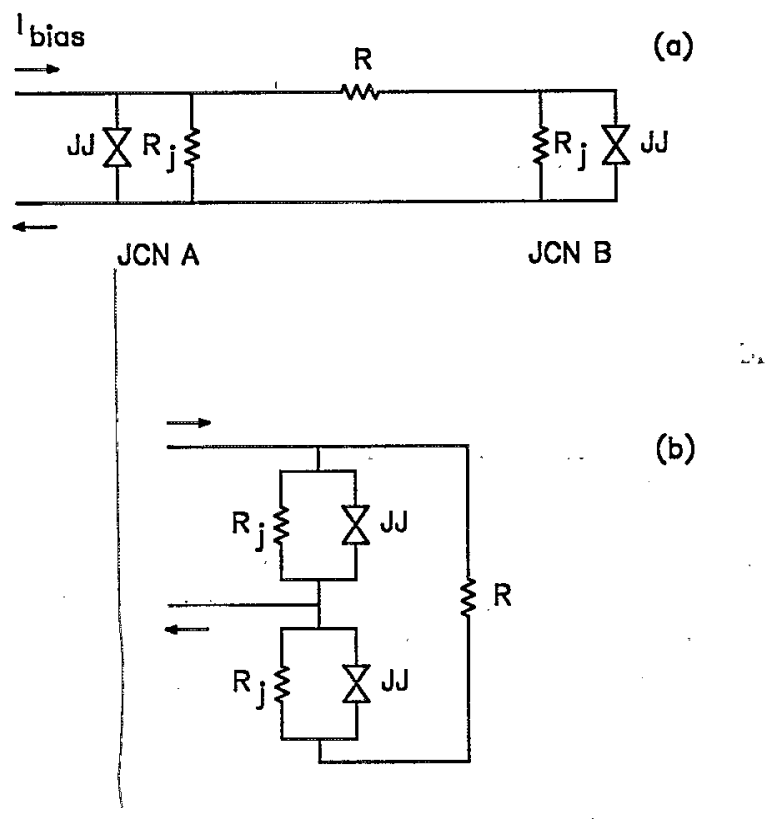

FIG. 1. Equivalent representations of a pair of resistively coupled Josephson junctions biased by a single current source.

peated as Fig. 2(a) in Ref. 6]. Inspection of these experimental curves confirms that they possess essentially the same features as our numerical results. The quoted values ${ }^{5}$ for junction resistance $(0.30 \Omega)$ and load resistance $(0.13$ $\Omega$ ) correspond to a coupling coefficient $\alpha=2.31$, rather close to the choices made for the numerical simulations in this study. The authors of Ref. 5 commented, “... with $I_{B}$ set to zero... no voltage locking occurs except for the uninteresting case of zero voltage." As we will now demonstrate, harmonic locking over a finite range of bias current should occur for this magnitude of $\alpha$ and may, in fact, be present in their experimental current to voltage $(I-V)$ curves, not an entirely uninteresting result after all.

A much more informative representation of the data is obtained by plotting normalized bias current versus the ratio $\left\langle d \phi_{B} / d t\right\rangle /\left\langle d \phi_{A} / d t\right\rangle$. Because dc junction voltage is proportional to $\langle d \phi / d t\rangle$, this ratio also equals

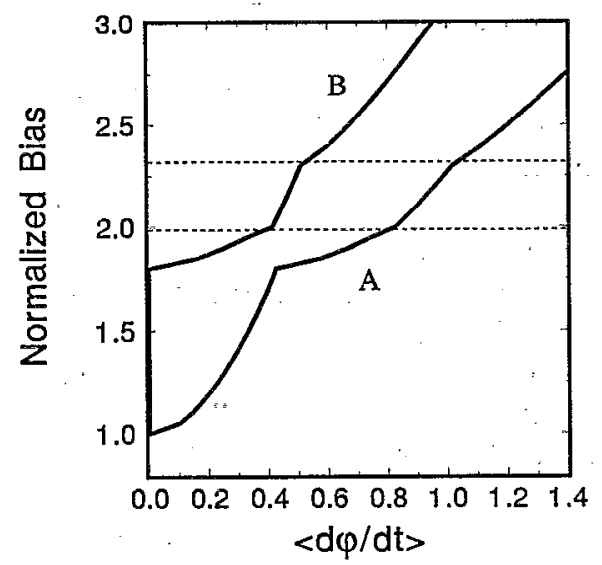

FIG. 2. Normalized bias current vs time-averaged phase velocity for junctions $A$ and $B$ with $\alpha=2.00$.

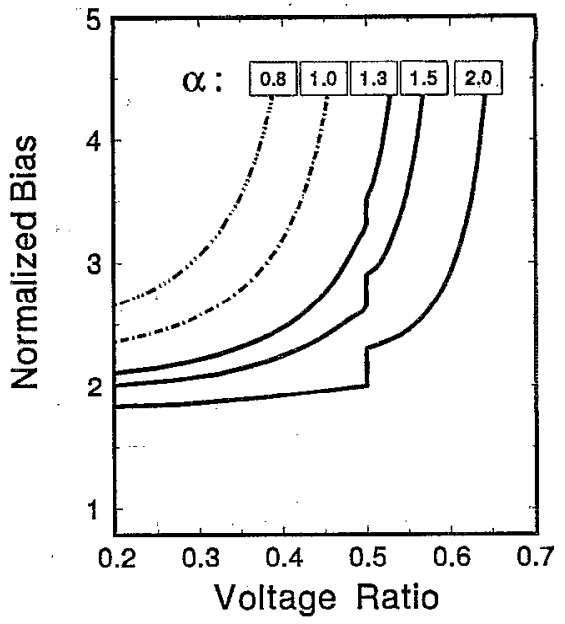

FIG. 3. Normalized bias current vs the ratio of the time averages of the junction voltages for several values of the coupling coefficient $\alpha$.

$\left\langle V_{J B}\right\rangle /\left\langle V_{J A}\right\rangle$. Plots of this type are shown in Fig. 3 for various choices of the coupling coefficient, including $\alpha=2.00$. It is immediately apparent that the region between the dotted lines in the previous figure is transformed now into a vertical line segment at 0.5 . In other words, the junction de voltages maintain a 1:2 relationship over this extended interval of bias current, a sign that there is harmonic locking ${ }^{2,13}$ of the junction oscillations. Figure 3 also reveals that $1: 2$ harmonic locking is present for all coupling coefficients greater than unity. For each curve there is a vertical asymptote which is approached for $I_{\text {bias }}^{*}>1$. This can casily be understood by realizing that, since the supercurrent channels in the Josephson junctions can never carry more than $I_{c}$, almost all of the current at very large bias must be carried by the resistive channels of the junctions. In this limit the circuit then acts like a simple voltage divider, and the asymptote is given by $\left\langle V_{J B}\right\rangle /\left\langle V_{J A}\right\rangle \rightarrow R_{J} /\left(R_{J}+R\right)=\alpha /(\alpha+1)$. Clearly this ratio is less than 0.5 for $\alpha<1$, and so $1: 2$ harmonic locking cannot occur for coupling coefficients less than unity. Equally obvious is the fact that $\alpha /(\alpha+1)$ is always less than 1 for finite $\alpha$; hence direct 1:1 locking can never be achieved in this system. This is a consequence of the fact that only a single external bias is employed (or equivalently that a second bias is set to zero).

Plots of $\left(\phi_{A}-2 \phi_{B}\right)$ versus normalized time are shown in Fig. 4 for $\alpha=2.00$. The three bias values $(1.99,2.15$, 2.33) correspond to the bottom, middle, and top of the locking zone, respectively. During each complete cycle of $\left(\phi_{A}-2 \phi_{B}\right)$, the phase in junction $B$ advances by $2 \pi$, while the phase of junction $A$ advances by $4 \pi$, as expected from 1:2 harmonic locking. Additionally, as the figure reveals, there is a phase offset between the two junctions (see the parameter $\theta$ in Sec. II A) which ranges in magnitude from 0 to $\pi$ over the extent of the locking zone.

\section{A. Locking range}

It is possible to estimate the size of the harmonic locking zone by the following approximation method. We begin 


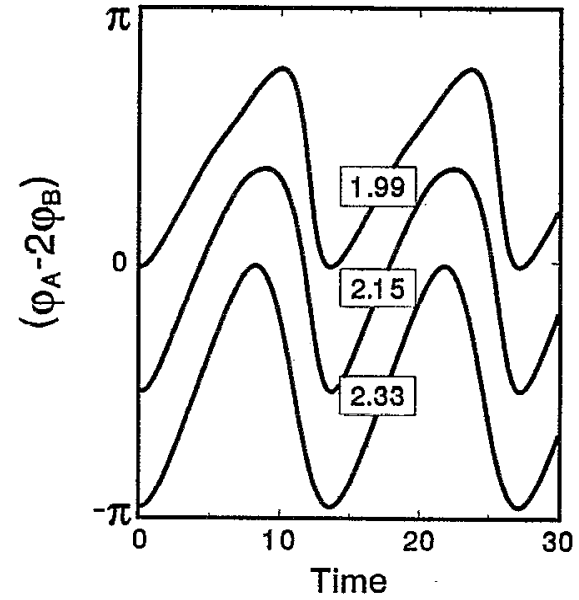

FIG. 4. Time dependence of the phase difference $\left(\phi_{A}-2 \phi_{B}\right)$ for $\alpha=2.00$ at three values of the normalized bias (shown in boxes). The horizontal range of $\mathbf{3 0}$ dimensionless time units is correct for the middle curve. The lower and upper curves have been stretched and compressed, respectively, as a viewing aid; the lower curve actually spans 26.8 normalized time units, while the upper curve covers 33.8 .

by noting that the normalized coupling energies of the two Josephson junctions are given by $H_{A}=1-\cos \left(\phi_{A}\right)$ and $H_{B}=1-\cos \left(\phi_{B}\right)$. Taking time derivatives of these energies and combining the resulting expressions with Eqs. (1) and (2), we obtain the energy flow into each of the two devices as

$$
\begin{aligned}
& \dot{H}_{A}=-(1+\alpha) \dot{\phi}_{A}^{2}+I_{\text {bias }}^{*} \dot{\phi}_{A}+\alpha \dot{\phi}_{A} \dot{\phi}_{B}, \\
& \dot{H}_{B}=-(1+\alpha) \dot{\phi}_{B}^{2}+\alpha \dot{\phi}_{A} \dot{\phi}_{B} .
\end{aligned}
$$

In the case of a periodic final state, the net flow of energy to each junction, taken over a complete cycle, must be zero, and hence

$$
\begin{aligned}
& \left\langle\dot{H}_{A}\right\rangle=-(1+\alpha)\left\langle\dot{\phi}_{A}^{2}\right\rangle+I_{\text {bias }}^{*}\left\langle\dot{\phi}_{A}\right\rangle+\alpha\left\langle\dot{\phi}_{A} \dot{\phi}_{B}\right\rangle=0, \\
& \left\langle\dot{H}_{B}\right\rangle=-(1+\alpha)\left\langle\dot{\phi}_{B}^{2}\right\rangle+\alpha\left\langle\dot{\phi}_{A} \dot{\phi}_{B}\right\rangle=0,
\end{aligned}
$$

where $\langle\cdot\rangle$ indicates an average over the period $T=2 \pi N /$ $\Omega$. Given $\alpha$, and assuming that the $\phi$ 's are known functions of time, Eqs. (5) and (6) can be solved for both $I_{\text {bias }}^{*}$ and the characteristic frequency $\Omega$. As a source of trial functions $\phi_{A}$ and $\phi_{B}$, consider the equation for an overdamped Josephson junction

$$
(1+\alpha) \dot{\phi}+\sin (\phi)=I_{\text {bias }}^{*},
$$

for which the analytic solutions are ${ }^{17}$

$$
\begin{aligned}
& \phi=\sin ^{-1}\left(I_{\text {bias }}^{*}\right), \text { for }\left|I_{\text {bias }}^{*}\right|<1, \\
& \phi=2 \tan ^{-1}\left[\frac{\sqrt{\left(I_{\text {bias }}^{*}\right)^{2}-1}}{I_{\text {bias }}^{*}} \tan \left(\frac{\Omega}{2} t\right)+\frac{1}{I_{\text {bias }}^{*}}\right], \\
& \quad \text { for }\left|I_{\text {bias }}^{*}\right|>1,
\end{aligned}
$$

where

$$
\Omega=\frac{1}{(1+\alpha)} \sqrt{\left(I_{\text {bias }}^{*}\right)^{2}-1}
$$

Hence

$$
\begin{aligned}
\phi= & 2 \tan ^{-1}\left[\frac{\Omega(1+\alpha)}{\sqrt{\Omega^{2}(1+\alpha)^{2}+1}} \tan \left(\frac{\Omega}{2} t\right)\right. \\
& \left.+\frac{1}{\sqrt{\Omega^{2}(1+\alpha)^{2}+1}}\right],
\end{aligned}
$$

and so

$$
\dot{\phi}=\frac{\Omega^{2}(1+\alpha)}{\sqrt{\Omega^{2}(1+\alpha)^{2}+1}+\cos (\Omega t)},
$$

where the time origin has been shifted by $\tan ^{-1}[\Omega(1+\alpha)]$. This type of function will now be used to construct trial solutions for the energy balance expressions (5) and (6). Specifically, we assume that the junction phases evolve as

$$
\dot{\phi}_{A}=\frac{\Omega^{2}(1+\alpha)}{\sqrt{\Omega^{2}(1+\alpha)^{2}+1}+\cos (\Omega t+\theta)}
$$

and

$$
\dot{\phi}_{B}=\frac{(\Omega / N)^{2}(1+\alpha)}{\sqrt{(\Omega / N)^{2}(1+\alpha)^{2}+1}+\cos \left(\frac{\Omega}{N} t\right)},
$$

where $\theta$ is the offset between the two phases, and $N$ is the ratio of the junction frequencies. It can be shown that

$$
\begin{aligned}
& \left\langle\dot{\phi}_{A}\right\rangle=\Omega, \quad\left\langle\dot{\phi}_{A}^{2}\right\rangle=\Omega \sqrt{\Omega^{2}+(1+\alpha)^{-2}} \\
& \left\langle\dot{\phi}_{B}\right\rangle=\Omega / N, \quad\left\langle\dot{\phi}_{B}^{2}\right\rangle=(\Omega / N) \sqrt{(\Omega / N)^{2}+(1+\alpha)^{-2}} .
\end{aligned}
$$

The energy-balance Eqs. (5) and (6) then yield

$$
\begin{aligned}
& 0=-\Omega \sqrt{\Omega^{2}(1+\alpha)^{2}+1}+\Omega I_{\text {bias }}^{*}+\alpha F(\theta, \Omega), \\
& 0=-(\Omega / N) \sqrt{(\Omega / N)^{2}(1+\alpha)^{2}+1}+\alpha F(\theta, \Omega),
\end{aligned}
$$

where

$$
F(\theta, \Omega)=\frac{\Omega}{2 \pi N} \int_{0}^{2 \pi N / \Omega} \dot{\phi}_{A} \dot{\phi}_{B} d t
$$

is the interaction energy between the junctions. For a given frequency $\Omega$ it is clear that $F$ has a maximum for $\theta=0$, and a minimum for $\theta=\pi$. By using these two limits, Eq. (10) can be solved for the range in $\Omega$ over which the two junctions will be locked in a frequency ratio of $1: N$. The range of $I_{\text {bias }}^{*}$ corresponding to this locking interval in $\Omega$ can then be determined from Eq. (9).

The perturbation treatment just described was used to predict locking ranges for the $N=2$ step, as shown in Fig. 5. This figure also displays data obtained from numerical solutions of the dynamical Eqs. (1) and (2). The agreement is generally quite good, particularly in view of the fact that the assumed trial functions [Eqs. (7) and (8)] are derived from the ideal behavior of isolated Josephson junctions and contain no mixing terms. 

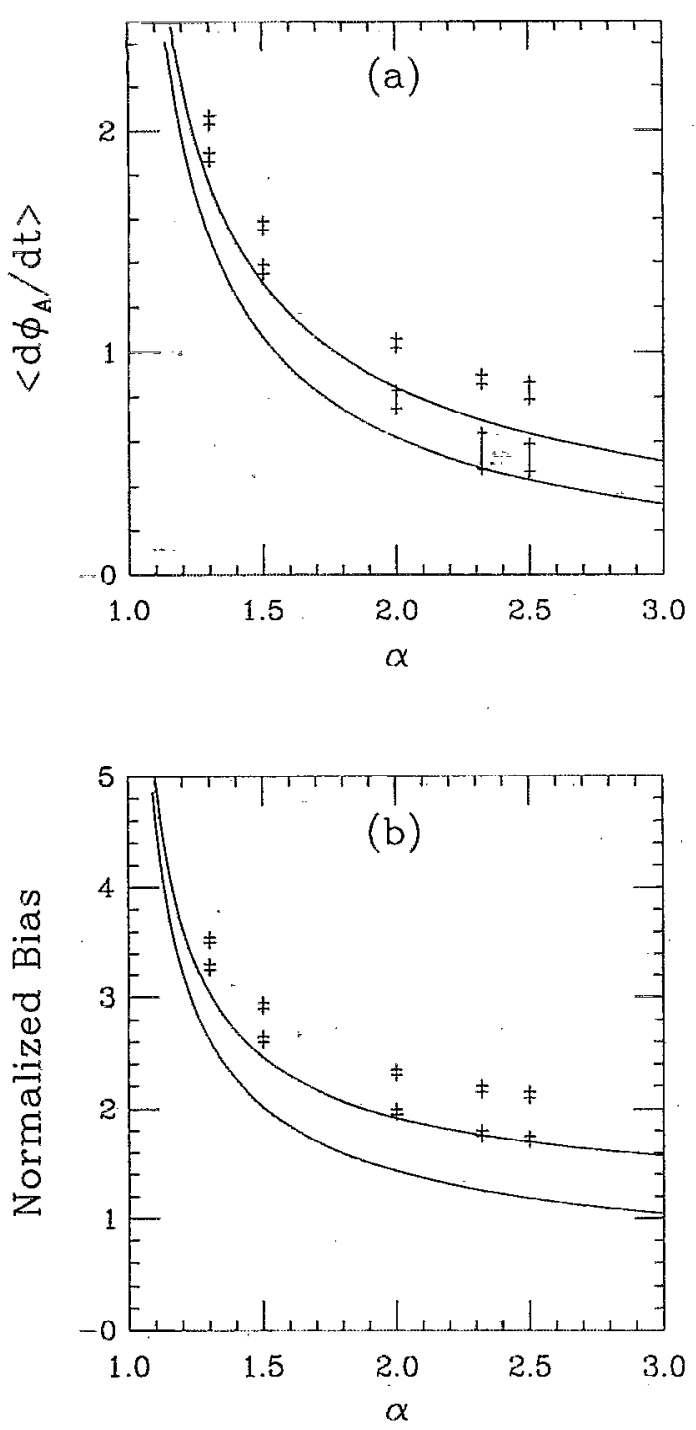

FIG. 5. Lower and upper limits of the 1:2 harmonic locking zone from perturbation analysis. (a) $\left\langle d \phi_{A} / d t\right\rangle$ or, equivalently, $\Omega$, as a function of the coupling coefficient $\alpha$. The locking zone is the region between the bounding curves. Numerically determined lower and upper limits for certain values of $\alpha$ are indicated by + symbols (together with the range of uncertainty). (b) Bias current at the lower and upper limits of the locking zone. Again, + symbols indicate numerically determined values at specific coupling coefficients.

\section{HARMONIC LOCKING: NONIDENTICAL JUNCTIONS}

The results presented in Sec. II A demonstrate that strong harmonic locking takes place between coupled identical Josephson junctions, at least for the $1: 2$ ratio. In reality, no two junctions are identical, so it is of interest to consider the effects of variations in $R_{J}$ and/or $I_{c}$. Thus, we define $\delta_{c} \equiv I_{C B} / I_{C A}$ as the ratio of the junction critical currents, and $\delta_{R} \equiv R_{J A} / R_{J B}$ as the ratio of junction resistances. Equations (1) and (2) become

$$
I_{\text {bias }}^{*}=(1+\alpha) \dot{\phi}_{A}-(\alpha) \dot{\phi}_{B}+\sin \left(\phi_{A}\right)
$$

and

$$
0=\left(\delta_{R}+\alpha\right) \dot{\phi}_{B}-(\alpha) \dot{\phi}_{A}+\delta_{c} \sin \left(\phi_{B}\right),
$$
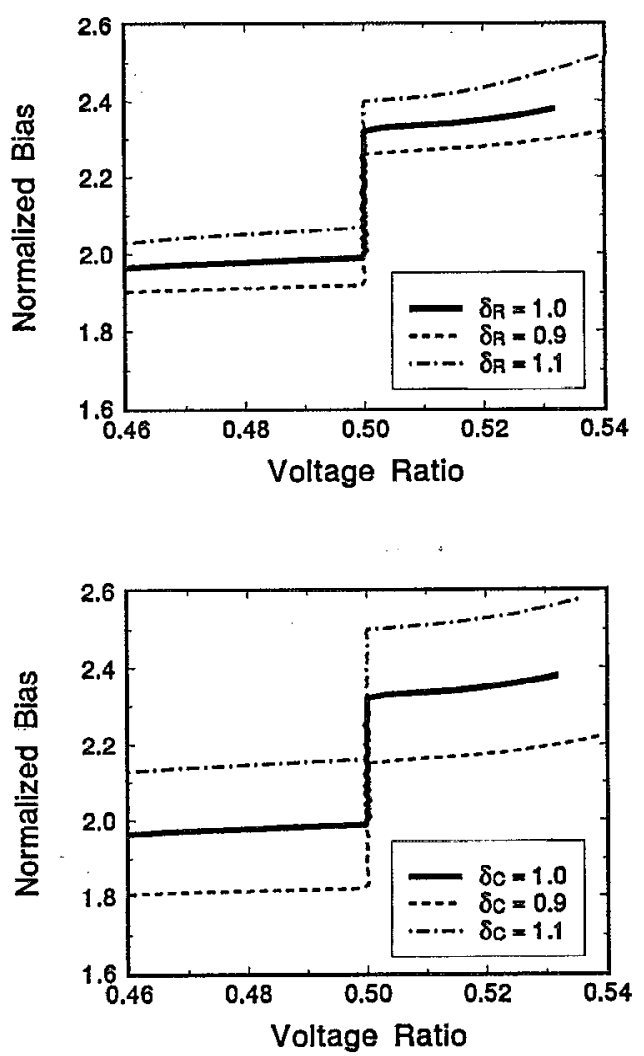

FIG. 6. Portion of $I_{\text {bios }}^{*}$ vs voltage ratio for $\alpha=2.00$ centered around the $1: 2$ harmonic locking zone. Upper plot: effect of unequal junction resistances. Lower plot: effect of unequal junction critical currents.

where $\alpha$ is now $R_{J A} / R$ and $I_{\text {bias }}^{*}$ is $I_{\text {bias }} / I_{c A}$. For ease of exposition, we shall consider one or the other, but not both, of these parameters as being different from unity.

(i) $\delta_{c}=1 ; \delta_{R} \neq 1$ : As shown in the upper section of Fig. 6 , inequality of the junction resistors induces small vertical shifts in the 1:2 harmonic locking zone, but does not radically alter the size of the locking region.

(ii) $\delta_{c} \neq 1 ; \delta_{R}=1$ : Computed 1:2 harmonic locking zones are shown in the lower section of Fig. 6 for the equal-junction case $\delta_{c}=1$, as well as for $\delta_{c}=0.90$ and 1.10 . Slightly larger vertical shifts in the locking zone result from junction asymmetry with respect to critical currents, but again the size of the locking region is not affected significantly.

Nerenberg et al. observed 2:1 harmonic locking in a double biasing arrangement (Ref. 13, Fig. 2) for a normalized input current range of approximately 1.65-1.70, a relative span of about $3 \%$. Their calculations, it should be noted, were done with parameters equivalent to somewhat weaker coupling but stronger junction asymmetry. For the single bias circuit under consideration here, with $\alpha>2.0$, Fig. 5 shows that relative locking intervals in excess of about $16 \%$ can be achieved.

Finally, we note that Nerenberg et al. ${ }^{13}$ also observed 3:1 harmonic locking. For identical junctions there is no evidence of any structure in our numerical data (Fig. 3) at the expected voltage ratio of 0.3333 . However when junction asymmetry is imposed, as illustrated in Fig. 7 ( $\alpha=1.5$, 


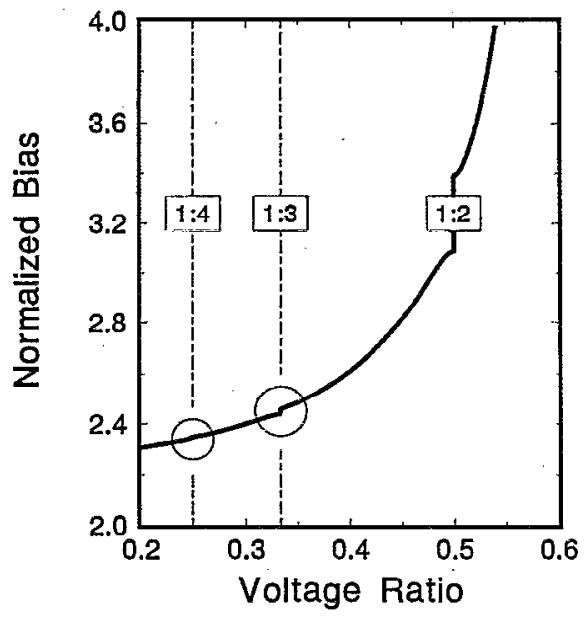

FIG. 7. Evidence of additional zones of harmonic locking when there is junction asymmetry: $\alpha=1.50, \delta_{c}=1.20$.

$\delta_{c}=1.20$ ), 1:3 locking does take place. In addition, a step at $1: 4$ is also just detectable.

\section{SUBHARMONIC OSCILLATIONS}

By analogy with the phenomenon of harmonic locking, $m: n$ subharmonic coherence ${ }^{15}$ between two Josephson oscillators consists of the phase of one junction advancing by $m(2 \pi)$ radians in a time interval during which the phase of the other junction advances by $n(2 \pi)$ radians. The ratio of time-averaged junction voltages is then $m / n$. A particular case of 3:5 coherence was discussed in Ref. 16. Since 3/5 $=0.6$, this specific example arose from a bias point located above the 1:2 harmonic locking zone. It was also demonstrated with respect to this operating point ${ }^{16}$ that the power spectra for the two junctions were composed primarily of a pair of dominant lines whose frequencies maintained this same 3:5 ratio.

We extend these observations to bias points below the 1:2 locking zone by choosing as a particular example $\alpha=1.00, I_{\text {bias }}^{*}=3.188$. This value of normalized bias was selected because it corresponds to a quite simple voltage ratio of $0.40=2 / 5$ for the $\alpha=1.00$ curve in Fig. 3. The junction wave forms shown in Fig. 8 may be compared to analogous wave forms from two completely noninteracting junctions biased separately so as to yield the same timeaveraged voltages as in the interacting case. As the figure demonstrates, the coupling provided by $\alpha=1.00$ is sufficient to significantly distort the wave forms. It also illustrates the source of the deviation between the perturbation and numerical results, as discussed in Sec. II. Power spectra of these junction oscillations, shown in Fig. 9, confirm the 2:5 relationship for the two principal frequencies (in normalized units) $f_{B}=0.12$ and $f_{A}=0.30$.

Although such data verify that, at particular bias values, the junction oscillations maintain a subharmonic relationship, there remains a question of whether this coherence holds over finite intervals of bias current. For the double-bias configuration, Frank et al. ${ }^{15}$ found a 2:3 lock-

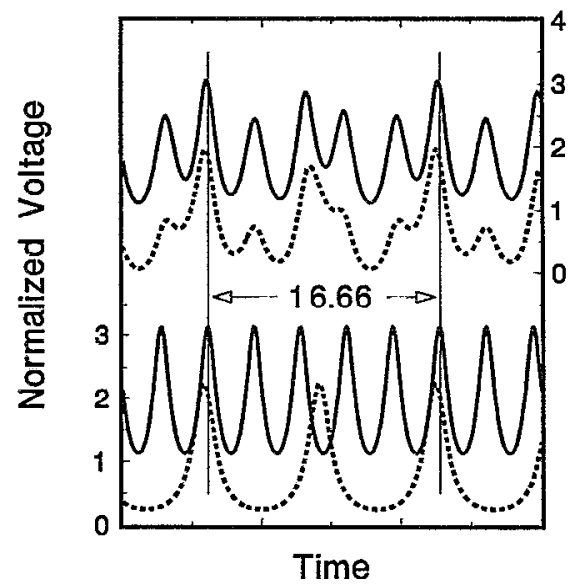

FIG. 8. Upper pair: wave forms of junction $A$ (solid) and junction $B$ (dotted) at $I_{\mathrm{bias}}^{*}=3.188, \alpha=1.00$. Lower pair: wave forms of two isolated junctions with $I_{\text {bias }}^{*}=2.1338$ (solid) and 1.2524 (dotted). These bias currents were chosen so that the upper and lower solid wave forms have the same dc value, as do the upper and lower dotted wave forms.

ing zone, although it was extraordinarily narrow, with a relative width of only about one part in ten thousand. In the present system for $\alpha=1.00$ and at $I_{\text {bias }}^{*}=3.188$, as discussed in the preceding paragraph, a 2:5 coherence is observed. But when the region immediately surrounding this
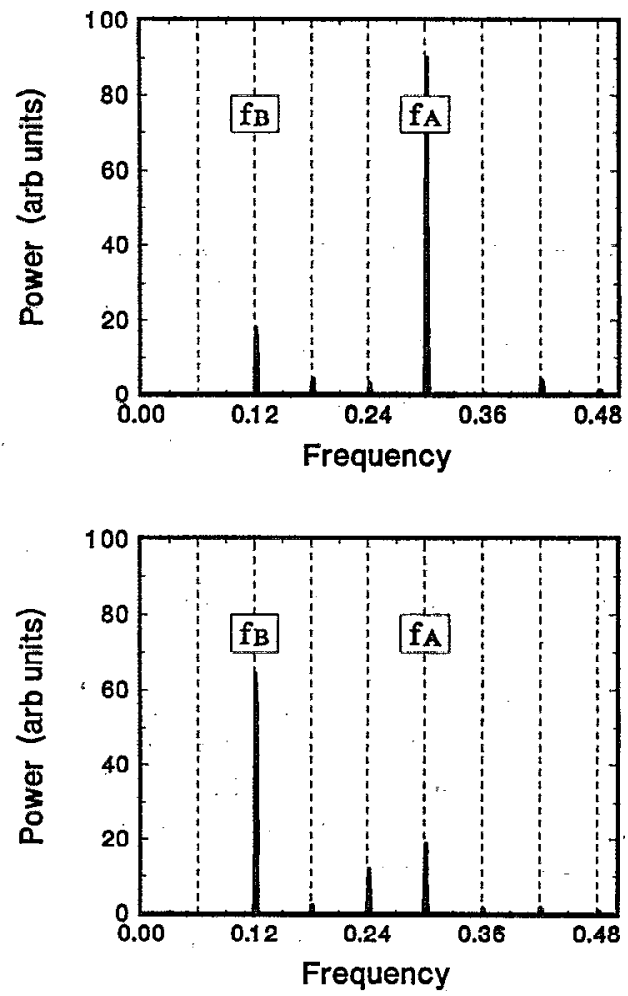

FIG. 9. Power spectra of the junction wave forms illustrating $2: 5$ subharmonic synchronization which occurs at $I_{\text {bias }}^{*}=3.19$ with $\alpha=1.00$. The upper plot applies to junction $A$, while the lower plot is for junction $B$. The dominant frequencies $f_{B}$ and $f_{A}$ exhibit the same 2:5 ratio as do the time averaged junction voltages. 


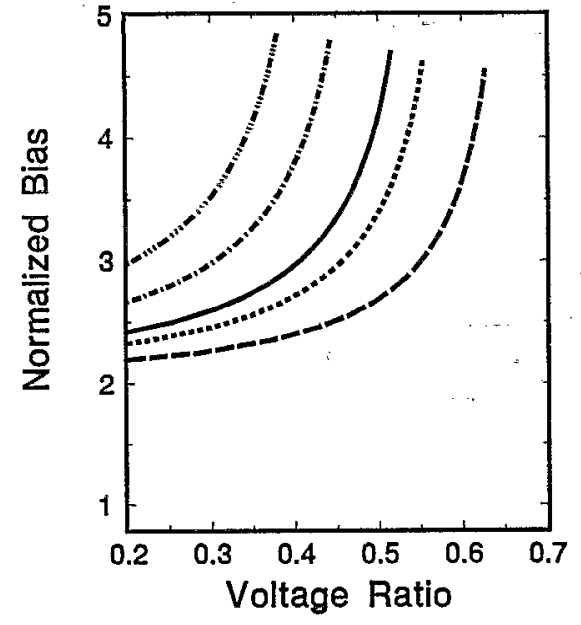

FIG. 10. Normalized bias current vs the ratio of the time-averaged junction voltages, $\left\langle V_{B}\right\rangle /\left\langle V_{A}\right\rangle$, calculated with only dc activated coupling between the junctions. Each curve is associated with a particular value of the coupling coefficient $\alpha$, the sequence of values being the same as shown in Fig. 3 (i.e., $\alpha=0.8,1.0,1.3,1.5$, and 2.0 , reading from left to right).

bias point was examined, even at 50 times the magnification of Fig. 3, no associated subharmonic locking zone could be seen.

Thus it is perhaps not unreasonable to speculate that by increasing or decreasing bias current (thereby moving up or down an $I-V$ characteristic), the junctions slide smoothly through an essentially continuous range of subharmonic frequency relationships. Some insight into this process can be gained by considering the behavior of the system when only dc coupling effects are included. The time-averaged current-voltage characteristics for each of the Josephson junctions are described by the usual expressions $^{17}$

$$
\begin{aligned}
& \left\langle V_{J A}\right\rangle=\sqrt{\left(\left\langle I_{J A}\right\rangle^{2}-1\right)}, \\
& \left\langle V_{J B}\right\rangle=\sqrt{\left(\left\langle I_{J B}\right\rangle^{2}-1\right)},
\end{aligned}
$$

where currents are normalized to $I_{c}$ and voltages to $I_{c} R_{j}$. Kirchhoff's node and loop conditions applied to the circuit arrangement (Fig. 1) require

$$
\begin{aligned}
& I_{\text {bias }}^{*}=\left\langle I_{J A}\right\rangle+\left\langle I_{J B}\right\rangle \\
& \left\langle V_{J A}\right\rangle=\left\langle V_{J B}\right\rangle+\left\langle I_{J B}\right\rangle / \alpha .
\end{aligned}
$$

For given $\alpha$, these four equations can be used to find $\left\langle V_{J_{A}}\right\rangle$ and $\left\langle V_{J B}\right\rangle$ and, thus, $\left(\left\langle V_{J B}\right\rangle /\left\langle V_{J A}\right\rangle\right)$ as functions of the input bias current $\Gamma_{\text {bias. }}^{*}$. The results, displayed in Fig. 10, are quite similar to the "exact" characteristics (Fig. 3) which are based on the complete system equations and, thus, include both ac and dc coupling. Of course, harmonic locking zones do not arise from this calculation since ac interactions between the junctions have been ignored.
This situation is reminiscent of the case of two junctions coupled by a transmission line ${ }^{9,16}$ where it was found that the reactive components of the line merely induced additional structure (decorations) onto underlying characteristics that were determined by the purely resistive link between the junctions. Considering Figs. 3 and 10, we now conclude that the characteristics associated with just a resistive link can themselves be reduced to underlying curves, shaped only by dc interjunction feedback and upon which ac effects (such as locking zones) are superimposed.

\section{CONCLUDING REMARKS}

It has been demonstrated that 1:2 harmonic locking between shunted Josephson junctions is very readily achieved and is not particularly sensitive to exact values of coupling coefficients, junction resistances, or critical currents. The sample configuration depicted in Fig. 1(a) or 1 (b) can be viewed as a "module" having an electrical common, an input terminal (bias current), and two output terminals (junction voltages). This suggests a possible application in which the module is intentionally biased within the harmonic locking zone, and the time-averaged output voltages, which maintain a precise 1:2 ratio, are used for relative calibrations such as linearity tests of voltmeters.

\section{ACKNOWLEDGMENTS}

Financial support was provided by the Natural Sciences and Engineering Research Council of Canada. Portions of this work were carried out under the auspices of the U.S. Department of Energy.

${ }^{1}$ J. Bindslev Hansen and P. E. Lindelof, Rev. Mod. Phys. 56, 431 (1984).

${ }^{2}$ A. K. Jain, K. K. Likharev, J. E. Lukens, and J. E. Sauvageau, Phys. Rep. 109, 309 (1984).

${ }^{3}$ C. Varmazis, R. D. Sandell, A. K. Jain, and J. E. Lukens, Appl. Phys. Lett. 33, 357 (1978).

${ }^{4}$ D. W. Jillie, Physica B 107, 741 (1981).

${ }^{5}$ D. G. McDonald and N. V. Frederick, Appl. Phys. Lett. 47, 530 (1985).

${ }^{6}$ D. G. McDonald, J. Appl. Phys. 60, 3247 (1986).

${ }^{7}$ K. Saitoh and T. Nishino, Phys. Rev. B 44, 7070 (1991).

${ }^{8}$ A. Davidson, IEEE Trans. Magn. MAG-17, 103 (1981).

${ }^{9}$ J. A. Blackburn, H. J. T. Smith, and N. Grønbech-Jensen, J. Appl. Phys. 70, 2395 (1991).

${ }^{10}$ N. Grønbech-Jensen, J. A. Blackburn, B. A. Huberman, and H. J. T. Smith, Phys. Lett. A 172, 131 (1992).

${ }^{11}$ Y. Imry and P. M. Marcus, IEEE Trans. Mag. MAG-13, 868 (1977).

${ }^{12}$ Y. Imry and L. S. Schulman, J. Appl. Phys. 49, 749 (1978).

${ }^{13}$ M. A. H. Nerenberg, J. A. Blackburn, and D. W. Jillie, Phys. Rev. B 21, 118 (1980).

${ }^{14}$ A. S. Deakin and M. A. H. Nerenberg, Phys. Rev. B 25, 1559 (1982).

${ }^{15}$ G. W. Frank, A. S. Deakin, M. A. H. Nerenberg, and J. A. Blackburn, Phys. Rev. B 35, 3138 (1987).

${ }^{16}$ H. J. T. Smith, J. A. Blackburn, and N. Grønbech-Jensen, J. Appl. Phys. 74, 5101 (1993).

${ }^{17}$ A. Barone and G. Paterno, Physics and Applications of the Josephson Effect (Wiley, New York, 1982), pp. 126-127. 